\title{
FORMANDO SEMILLEROS DE INVESTIGACIÓN QUE TRABAJAN POR LA INCLUSIÓN
}

\section{TRAINING RESEARCH SEEDBEDS THAT WORK FOR INCLUSION}

\author{
Bertha Alice Naranjo Sánchez ${ }^{1}$ - William \\ Andrés Villavicencio Benalcázar², \\ Universidad Politécnica Salesiana, Docente \\ Investigadora, Guayaquil, Ecuador,
}

Azucena Del Rocío Naranjo Sánchez ${ }^{3}$,

Ministerio de Educación, Guayaquil, Ecuador

\section{RESUMEN}

La sociedad demanda investigación, pues es la única forma técnica científica de resolver problemas que no tienen respuestas hasta el día de hoy, por ello es necesario fortalecer los procesos de investigación en los jóvenes con el

$1 \quad$ Bertha Alice Naranjo Sanchez. Docente e Investigadora de la Universidad Politécnica Salesiana Sede Guayaquil

https://orcid.org/0000-0002-4386-2335

bnaranjo@ups.edu.ec

$2 \quad$ Universidad Politécnica Salesiana, Estudiante Investigador, Guayaquil, Ecuador, wvillavicenciob@est.ups. edu.ec

https://orcid.org/0000-0003-3023-066X

3 Docente Investigadora, Guayaquil, Ecuador, azucena.naranjo@educacion.gob.ec

https://orcid.org/0000-0003-3423-1870 objetivo de despertar el interés y la motivación hacia la ciencia. Este artículo describe el proceso de investigación formativa que el grupo de investigación denominado Tecnología de Información y Comunicación asociado a discapacidad desarrolla para hacer efectiva la transferencia de competencias en materia de investigación a estudiantes del semillero de la Universidad Politécnica Salesiana, de las Carreras de Ingeniería de Sistemas y Computación, de la sede Guayaquil.

\section{PALABRAS CLAVE}

Investigación, semilleros, investigación formativa, competencias, inclusión. 


\section{ABSTRACT}

Society demands research, because it is the only scientific technical way to solve problems that do not have answers until today, so it is necessary to strengthen the research processes in young people with the aim of raising interest and motivation towards the science. This article describes the formative research process that the research group called Information and Communication Technology associated with disability develops to make effective the transfer of research skills to students of the Salesian Polytechnic University, of Systems and Computer Engineering Careers, from Guayaquil.

\section{KEYWORDS}

Research, seedbeds, formative research, skills, inclusion.

\section{INTRODUCCIÓN}

En el año 2013, en la Universidad Politécnica Salesiana (UPS), Carrera de Sistemas surgió el grupo de investigación "Tecnología de Información y Comunicación asociado a discapacidad" (TICAD) como una alternativa para atender una necesidad existente en la sociedad, la inclusión educativa de estudiantes con discapacidad (Naranjo, Naranjo, Mora, \& Huilcapi , 2015). Esta necesidad motivó al grupo TICAD a investigar los aspectos de la inclusión educativa con el objetivo de lograr la efectividad de este proceso en el aula, así se revisó la documentación existente hasta esa fecha, se revisaron las investigaciones previas relacionadas con el tema y se destinaron esfuerzos para identificar los elementos básicos (Naranjo, 2017, 2018) que permitirían adecuar la Universidad para la inclusión de personas con discapacidad (PCD).

Una de las necesidades claves de las personas con discapacidad es el acceso a productos adaptados o Tecnologías de información y comunicación (TIC) de apoyo. Estos dispositivos en su mayoría tienen un costo alto, lo cual resulta inasequible o en su defecto no existen en el mercado local. Ante esta situación los estudiantes de la Carrera de Ingeniería de Sistemas tomaron la iniciativa de formar grupos de trabajo que permitan ofrecer a través de la investigación soluciones en materia de TIC para personas con discapacidad, naciendo así el semillero de investigación del grupo TICAD, ente encargado de responder a las demandas de la sociedad y de los estudiantes con discapacidad, a través de la aplicación de procesos de investigación en los que las necesidades basadas en TIC adaptativas o tecnologías de apoyo podrían ser cubiertas.

En la UPS Sede Guayaquil no existían semilleros de investigación, por lo que su creación fue una novel actividad en la que el grupo TICAD incursionó, acompañando a ello la necesidad de desarrollar un proceso de investigación formativa que permita a los estudiantes vivir la experiencia de hacer investigación en temas de inclusión y discapacidad enmarcados en las áreas temáticas que el grupo mantiene.

El semillero se convirtió en una opción formativa que permite fortalecer las competencias en materia de investigación en forma adicional a la ya existente asignatura de investigación presente en la malla curricular, es la opción extracurricular que el grupo de investigación ofrece para que los estudiantes pueden aprender a investigar en la práctica.

En este artículo se describe el proceso de investigación formativa que los estudiantes del semillero de investigación transitan con el fin de desarrollar sus competencias en investigación enmarcadas en áreas temáticas de inclusión y discapacidad.

\section{MARCO TEÓRICO}

Las estadísticas revelan resultados desalentadores en los principales indicadores 
de las actividades de ciencia, tecnología e innovación en el Ecuador. Un estudio realizado por el INEC en el periodo 2009 -2011 muestran que apenas el $7,4 \%$ de las empresas en el país introdujo un bien completamente nuevo al mercado (INEC, 2012), mientras que el análisis realizado por la misma entidad para el rango comprendido entre los años 2012-2014 permite identificar un decremento del $2 \%$ de empresas que produjeron un bien nuevo con respecto al periodo de evaluación anterior esto es del 2009 al 2011 (INEC, 2015), entendiéndose como producto nuevo "aquel cuyas características fundamentales difieren significativamente de todos los productos previamente producidos por la empresa" (OCDE, 2005), lo que evidencia la necesidad de fortalecer la investigación con el fin de proponer soluciones o innovaciones para la sociedad.

El objetivo 10 del Plan Nacional del Buen Vivir propuso "Impulsar la transformación de la matriz productiva", y a través del numeral 2) se estableció "Promover la intensidad tecnológica en la producción primaria, de bienes intermedios y finales" lo que guarda relación con lo indicado en el objetivo 4) "Fortalecer las capacidades y potencialidades de la ciudadanía" que en el numeral 6) expresa el compromiso gubernamental de "Promover la interacción recíproca entre la educación, el sector productivo y la investigación científica y tecnológica, para la transformación de la matriz productiva y la satisfacción de necesidades", estableciendo para ello en el literal h) "Impulsar políticas, estrategias, planes, programas 0 proyectos para la investigación, el desarrollo y la innovación ( $(+D+i)$ de tecnologías de información y comunicación" (SENPLADES, 2009, 2013).

Además, en los planes estratégicos del gobierno del 2009 al 2013, y en el del 2013 al 2017, se resalta que entre otros temas existen brechas de atención en temas de discapacidad (SENPLADES, 2009, 2013), por lo que estos dos temas, tanto investigación como discapacidad pueden y deben ser tratados en corresponsabilidad por las Universidades, las cuales regularmente incorporan el tema de la investigación en la malla curricular de las Carreras con las asignaturas metodología de la investigación y seminario de investigación, mientras se sigue ejerciendo la docencia como actividad primaria en el aula.

Fomentar las vocaciones en investigación para proveer a la sociedad de jóvenes investigadores que puedan generar descubrimientos significativos que permitan resolver los problemas que nos aquejan, debe ser responsabilidad de las Universidades y en especial de la academia, pero esta tarea no es fácil y debe ser estructurada con el fin de poder alcanzar dicho objetivo. El docente debe preparar entonces en la academia a los estudiantes para la investigación y una forma alternativa la constituye los semilleros de investigación.

Revisando aspectos de la investigación formativa en la educación superior y en especial de los semilleros, encontramos algunos aportes de trabajos previos que se describen en la tabla 1. 
Tabla 1: Aportes de la investigación formativa y los semilleros

\begin{tabular}{|c|c|c|c|}
\hline Año & Artículo/Libro & Autores & Aportes \\
\hline 2014 & $\begin{array}{l}\text { La investigación } \\
\text { formativa a través } \\
\text { del aprendizaje } \\
\text { orientado a proyectos: } \\
\text { una propuesta de } \\
\text { innovación en el grado } \\
\text { de Pedagogía }\end{array}$ & $\begin{array}{l}\text { (Vilá Baños, Rubio } \\
\text { Hurtado, \& Berlanga } \\
\text { Silvente, 2014) }\end{array}$ & $\begin{array}{l}\text { El alumnado adopta un rol } \\
\text { activo, trabaja en equipo, } \\
\text { busca información, y planifica } \\
\text { el trabajo, tomando sus } \\
\text { propias decisiones, integrando } \\
\text { conocimientos, y desarrollando } \\
\text { competencias diversas. } \\
\text { El rol del profesorado es } \\
\text { acompañar a los estudiantes } \\
\text { hacia el aprendizaje } \\
\text { independiente, motivándolos a } \\
\text { trabajar de forma autónoma. }\end{array}$ \\
\hline 2016 & $\begin{array}{l}\text { Sentidos y } \\
\text { perspectivas } \\
\text { sobre Semilleros } \\
\text { de Investigación } \\
\text { Colombianos, } \\
\text { hacia la lectura de } \\
\text { una experencia } \\
\text { Latinoamericana. }\end{array}$ & $\begin{array}{l}\text { (Gallardo Cerón, } \\
\text { 2016) }\end{array}$ & $\begin{array}{l}\text { Describe los Sentidos y } \\
\text { perspectivas de los Semilleros } \\
\text { de Investigación, su formación } \\
\text { y necesidades. En los } \\
\text { Semilleros de Investigación } \\
\text { la investigación formativa } \\
\text { emerge con la participación del } \\
\text { estudiante investigador actor y } \\
\text { constructor. } \\
\text { Los semilleros se convierten } \\
\text { en estructuras alternativas } \\
\text { que impactan en la comunidad } \\
\text { científica. }\end{array}$ \\
\hline 2017 & $\begin{array}{l}\text { La investigación } \\
\text { formativa universitaria } \\
\text { ¿Posibilidad o } \\
\text { necesidad? }\end{array}$ & (Velázquez, 2017) & $\begin{array}{l}\text { La investigación formativa } \\
\text { es un subcomponente } \\
\text { procesal que aúna esfuerzos } \\
\text { de la investigación con la } \\
\text { intención de crear nuevo } \\
\text { conocimiento a partir del } \\
\text { desarrollo de proyectos de } \\
\text { investigación desarrollando } \\
\text { no sólo competencias básicas } \\
\text { y específicas sino hasta las } \\
\text { investigativas, por lo que es } \\
\text { una necesidad del propio } \\
\text { desarrollo socioeducativo. }\end{array}$ \\
\hline
\end{tabular}




\begin{tabular}{|c|c|c|c|}
\hline 2017 & $\begin{array}{l}\text { Uso de recursos y } \\
\text { herramientas TIC } \\
\text { para el fortalecimiento } \\
\text { de competencias } \\
\text { investigativas } \\
\text { en el semillero } \\
\text { de investigación } \\
\text { del programa de } \\
\text { Licenciatura en } \\
\text { Informática de la } \\
\text { Universidad de Nariño }\end{array}$ & $\begin{array}{l}\text { (Paz Saavedra, } \\
\text { Guerrero, \& Ortega, } \\
\text { 2017) }\end{array}$ & $\begin{array}{l}\text { Fortalecer las competencias } \\
\text { investigativas de los futuros } \\
\text { licenciados mediante el uso } \\
\text { de diferentes recursos y } \\
\text { herramientas TIC. }\end{array}$ \\
\hline 2019 & $\begin{array}{l}\text { Semillero de } \\
\text { investigación: } \\
\text { Estrategia educativa } \\
\text { para } \\
\text { promover la } \\
\text { innovación tecnológica }\end{array}$ & $\begin{array}{l}\text { (Cantú, Medina, \& } \\
\text { Martínez, 2019) }\end{array}$ & $\begin{array}{l}\text { Describe la práctica de la } \\
\text { Investigación extracurricular, } \\
\text { tanto para estudiantes como } \\
\text { para docentes, para la } \\
\text { generación y desarrollo de } \\
\text { proyectos tecnológicos y } \\
\text { prototipos funcionales }\end{array}$ \\
\hline
\end{tabular}

Elaborado por autores, a partir de las fuentes referidas en la tabla.

Como refiere la tabla 1 , los trabajos previos sobre investigación formativa y los semilleros coinciden en la necesidad de desarrollar las competencias de los estudiantes en temas de investigación.

Según (Parra, 2004) citado por (De la Ossa , Pérez , Patiño, \& Montes, 2012) indica:

La investigación formativa, base del proceso universitario, se define como una herramienta del proceso de enseñanza aprendizaje, no es otra cosa que la enseñanza a través de la investigación o enseñar usando el método científico para comprender fenómenos y aplicarlos a modelos conceptuales que fundamentan la calidad de los pregraduados y mantengan activos a los docentes investigadores y sus grupos científicos.

Para (Montoya \& Peláez, 2013) el concepto de investigación formativa:
Se usa para referirse a la capacidad que deben adquirir los estudiantes y profesores para emplear los métodos de investigación como estrategia de enseñanza aprendizaje. Su pretensión no es la construcción o exploración de nuevos conocimientos, como ocurre con la investigación científica, en este caso se espera que se emplee el método de investigación con el fin de desarrollar competencias para apropiar el conocimiento construido sobre un tema.

En estos conceptos se aprecia la aplicación de la investigación formativa dentro del proceso enseñanza-aprendizaje pero su implementación deber ir más allá del aula y de las asignaturas.

"El semillero es un ambiente diseñado para identificar y reafirmar la vocación investigativa en general o en un campo o área específica, está integrado por un investigador de carrera que lo lidera, y un grupo de estudiantes o profesores que desean iniciarse en la investigación" 
(Miyahira, 2009) citado por (Velázquez, 2017).

Estos enfoques coinciden en apoyar la teoría que la investigación formativa y los semilleros de investigación constituyen el espacio ideal para crear un entorno donde se desarrollen las competencias de los estudiantes en investigación.

Como lo indica (Jiménez, 2017):

Los semilleros de investigación, para esta época, surgen como respuesta a la necesidad de brindar a los estudiantes espacios académicos donde fortalezcan las dinámicas propias de la metodología de la investigación formativa. El semillero de investigación ofrece una serie de ventajas consustanciales debido a que, en este espacio, se viven de manera práctica los procesos de investigación, además de adquirir una serie de habilidades que diferencian a los estudiantes del semillero con relación a los estudiantes del común. En efecto, quienes integran el semillero desarrollan otro tipo de mentalidad con relación no solo a la investigación y sus desafíos, sino con relación a la universidad como un centro de producción de conocimiento.

Conocer el proceso formativo de estudiantes universitarios que trabajan bajo el enfoque de investigación formativa dentro del semillero del grupo TICAD es el objetivo que se plantea este artículo.

\section{METODOLOGÍA}

Para el desarrollo de este trabajo se utilizó la metodología descriptiva y explicativa, en la recolección del conocimiento existente y los trabajos previos que permiten explorar la investigación formativa y el contexto de los semilleros. Las técnicas usadas para el relevamiento de información del proceso formativo fueron: entrevista, cuestionario, análisis documental, focus group. Tres docentes investigadores del grupo TICAD fueron relevados a través de entrevistas, para identificar las estrategias y técnicas de aprendizaje usadas para fortalecer las competencias investigativas de los estudiantes de la Carrera de Ingeniería de Sistemas/Computación; 100 estudiantes del semillero de investigación del grupo TICAD que desarrollaron proyectos en asignaturas fueron consultados a través de un cuestionario para conocer la forma en que se trabaja al interior del semillero, criterios que fueron confirmados usando la técnica focus group con estudiantes representantes de los 3 cursos visitados. En el relevamiento del proceso de forma adicional se entrevistó al personal del Centro de apoyo para la inclusión (CAl) y se consultó la documentación existente en ese Centro y en la oficina de trabajo del grupo TICAD; se evidenciaron además en el sitio web institucional los eventos que el grupo organiza para el semillero y se consultaron las carpetas que mantiene en la nube, el grupo de investigación, para el proceso autoformativo de los estudiantes.

\section{RESULTADOS}

Luego de aplicar las técnicas de relevamiento de información descritas en la metodología encontramos que si bien el semillero surge de estudiantes que de forma voluntaria colaboraban con el grupo TICAD, la formalización del mismo dentro de la Universidad Politécnica Salesiana la establece el grupo de investigación, así surge el primer semillero de investigación en la Universidad Politécnica Salesiana sede Guayaquil en el año 2013, adherido a un grupo de investigación, con el objetivo de eliminar esa brecha existente entre la academia y la investigación, para fomentar el desarrollo de las competencias en investigación de los futuros profesionales de la carrera de Ingeniería en Sistemas y trabajar en forma específica en temas asociados a discapacidad e inclusión. 
Al 2019, el semillero mantiene 200 estudiantes participando de forma voluntaria y activa en los procesos de aprendizaje establecidos en este tipo de investigación formativa.

Al cursar asignaturas específicas, dentro de la academia, los estudiantes se pueden adherir voluntariamente al semillero, trabajando en proyectos de asignaturas e iniciando el desarrollo de un proceso investigativo dependiendo del área temática y grupo al cual se adhieren formalmente.

El semillero está formado por diversos grupos los cuales en función del nivel instruccional por el que transitan pueden crear adaptaciones/ productos de apoyo/tic asistidas, software educativo inclusivo, software de gestión administrativa, desarrollar artículos académicos o de investigación, proyectos, cursos en TIC inclusivas; sus productos resultantes son entregados a la sociedad, en especial a diversas instituciones de la Red Académica de Apoyo e Investigación en Tecnologías inclusivas (RAITI), tal como lo confirmaron en entrevista los delegados del CAI de la UPS Sede Guayaquil.

Estructuralmente el semillero cuenta con procesos establecidos, normas, políticas y procedimientos, guías e instructivos, definidos para una adecuada organización.

En función de los productos resultantes que ofrecen los diferentes grupos de semilleros, se clasifican en:

\section{Semillero de adaptaciones/productos de apoyo/TIC asistidas}

La labor de este semillero se desarrolla dentro de la academia y a él se adhieren los estudiantes que ejecutan adaptaciones de hardware, creando productos tecnológicos que faciliten la interacción de las PCD con el computador. Los productos de apoyo, tecnologías asistivas, entre otros dispositivos que produce este semillero están destinados a ser implementados en las instituciones de la red RAITI, algunos de sus prototipos se mantienen en exhibición en el CAI.

\section{Semillero de software educativo inclusivo}

El semillero de Software educativo inclusivo, provee de software hecho a la medida acorde a las necesidades educativas de las instituciones que pertenecen a la red RAITI el cual puede ser desarrollado en entornos web o en plataformas móviles, cumpliendo con principios de accesibilidad.

\section{Semillero de software de gestión administrativa}

Este semillero desarrolla software que facilita la operatividad de las instituciones de la red.

\section{Semillero de software de gestión académica}

El semillero crea los aplicativos necesarios para que las diversas instituciones educativas especiales que integran la red, puedan realizar el control y la gestión académica acorde a las necesidades de las mismas.

\section{Semillero de divulgación}

En este semillero los estudiantes aprenden sobre TIC inclusivas las cuales luego de un proceso de capacitación son transferidas a la sociedad en cursos gratuitos organizados por el grupo TICAD y dirigido a personas con discapacidad, docentes o instituciones de la red RAITI. Esta actividad les permite comprender la discapacidad y la inclusión, a la vez que obtienen el conocimiento necesario para poder continuar y adherirse en caso de ser de su interés al semillero de proyectos o de artículos.

\section{Semillero de proyectos}

Se dedica al desarrollo de proyectos, los cuales pueden ser implementados en instituciones educativas, asociaciones, federaciones $y$ fundaciones de personas con discapacidad. 


\section{Semillero de artículos}

Este semillero se dedica a la elaboración de artículos académicos o de investigación dentro de los proyectos que administra el grupo TICAD.

Las competencias profesionales de la Carrera así como las transversales con el eje de inclusión e investigación son trabajadas en los semilleros. En relación a las competencias de investigación, éstas se desarrollan en cuatro niveles formativos que son:

\section{Nivel principiante}

Apoya los procesos administrativos relacionados a investigación, reciben capacitación en materia de discapacidad e inclusión. Las competencias analizadas e interiorizadas en este nivel son temas como la metodología de investigación, código de ética del investigador, tipos y técnicas de investigación En el área de discapacidad e inclusión, aspectos como el glosario inclusivo y la normativa vigente son los que encierran el aprendizaje en la línea de investigación del grupo. Se fomentan valores como responsabilidad, honestidad, ética, se aplican principios y valores que deben evidenciarse en el trabajo diario.

\section{Nivel intermedio}

En este nivel los estudiantes desarrollan un producto tecnológico o de apoyo, realizan adaptaciones de hardware y facilitan la interacción hombre-máquina, aprenden las normas INEN del campo profesional de Ingeniería, normas de calidad y normas de accesibilidad entre otros conceptos asociados a la carrera.

\section{Nivel avanzado}

En este nivel los semilleristas desarrollan artículos académicos o proyectos de grado, pero deben haber aprobado satisfactoriamente conocimientos curriculares de lecto-escritura y metodología de investigación. En este nivel aplican métodos, técnicas e instrumentos de investigación, interactúan con herramientas de software estadístico, y participan como apoyo en proyectos de investigación del grupo.

\section{Nivel de madurez investigativa}

Este nivel lo alcanzan los estudiantes que han aprobado las competencias previas de los niveles anteriores, y en función de ello, pueden proponer y/o coordinar proyectos de investigación.

Dentro del semillero se trabaja con estrategias de aprendizaje como son: aprendizaje significativo y ABP (Aprendizaje basado en proyectos), entre las técnicas que más destacan para el desarrollo de proyectos y productos, se encuentran trabajo colaborativo y cooperativo.

El proceso formativo se complementa con el aprendizaje y uso de las TIC adaptativas/ asistidas, el entorno virtual disponible en la nube, que contiene material multimedia preparado por el grupo de investigación TICAD y material seleccionado que se utiliza en el proceso formativo de los semilleristas, así se produce un autoaprendizaje, el mismo que se complementa con reuniones, encuentros, cursos formativos y eventos presenciales, pero el proceso de mayor aprendizaje es en la práctica y trabajando con el grupo de investigación, en la oficina que el grupo mantiene, así en un entorno investigativo adecuado, el proceso de investigación formativa fluye para entregar a la sociedad un profesional competente y a la vez un potencial investigador.

Esta propuesta permite hacer efectivo el desarrollo de las competencias en materia de investigación para los estudiantes de las Carreras de Ingeniería de Sistemas y Computación. Se desarrolla de forma gradual, los conocimientos son transferidos a medida que los estudiantes van avanzando también académicamente en los niveles de estudio, indicaron los docentes 
entrevistados, destacando que esto permite materializar la investigación formativa.

En este proceso formativo de semilleristas participan investigadores del grupo TICAD, estudiantes, asociaciones, el CAI de la Universidad Politécnica Salesiana sede Guayaquil, la red RAITI integrada por federaciones, asociaciones y fundaciones de personas con discapacidad, todos trabajando por un mismo objetivo el cual es facilitar la autonomía de las personas con discapacidad, la atención a necesidades básicas enmarcadas en el derecho a la educación inclusiva y TIC asistivas.

\section{CONCLUSIONES}

En este artículo se describe el proceso que se sigue para desarrollar la investigación formativa de los semilleros de investigación del grupo TICAD, una experiencia educativa que nace en la academia, en la que los estudiantes aprenden a investigar haciendo investigación en la práctica, transitando por niveles de conocimiento para desarrollar competencias investigativas $y$ satisfaciendo a su vez necesidades puntuales en interacción con la sociedad, conectándose con la inclusión y discapacidad.

En este modelo de desarrollo de competencias para los estudiantes, en materia de investigación formativa se observan y destacan elementos importantes para trabajar desde el campo académico en el investigativo, creando espacios de trabajo en el semillero de investigación, definiendo esquemas de organización del semillero, estableciendo el proceso formativo, implementando metodologías y estrategias de trabajo, así como los niveles formativos que transitan los estudiantes investigadores, entre otros aspectos relevantes.

Las actividades, habilidades y competencias que desarrollan los estudiantes investigadores en los diferentes niveles permiten apreciar un modelo de desarrollo progresivo de las cualidades que un investigador debe tener, lo que permitirá entregar a la sociedad no sólo un profesional competente sino también un futuro investigador.

Los aportes de este trabajo permiten reflexionar en la necesidad de que la docencia se oriente hacia la formación de profesionales con competencias científicas, logrando que en el proceso de enseñanza - aprendizaje se fortalezcan las vocaciones científicas en los jóvenes universitarios.

\section{AGRADECIMIENTOS}

Al grupo de investigación TICAD, a la Universidad Politécnica Salesiana, a las Carreras de Ingeniería de Sistemas y Computación de la sede Guayaquil y a las instituciones de la red RAITI.

A los integrantes del semillero de investigación TICAD por brindar todas las facilidades $y$ cooperación en el relevamiento de información.

AI CAI de la UPS Sede Guayaquil por facilitarnos información relacionada a proyectos de los semilleros del grupo TICAD implementados en las instituciones de la red RAITI.

\section{REFERENCIAS BIBLIOGRÁFICAS}

Cantú, I., Medina, A., \& Martínez, F. (2019). Semillero de investigación: Estrategia educativa para promover la innovación tecnológica. Revista Ibeeroamericana para la investigación y el desarrollo educativo, 10(19).

De la Ossa , J., Pérez , C., Patiño, R., \& Montes, D. (2012). La investigación formativa como una necesidad en el pregrado. Revista Colombiana de Ciencia Animal, 1-3.

Gallardo Cerón, B. N. (2016). Sentidos $y$ perspectivas sobre Semilleros 
de Investigación Colombianos, hacia la lectura de una experencia Latinoamericana.

INEC. (2012). Recuperado el 28 de 08 de 2019, de https://www.ecuadorencifras.gob. ec/documentos/web-inec/Estadisticas_ Economicas/Ciencia_TecnologiaACTI/Presentacion_de_principales_ resultados_ACTI.pdf

INEC. (2015). Recuperado el 27 de 08 de 2019, de https://www.ecuadorencifras.gob. ec/documentos/web-inec/Estadisticas Economicas/Ciencia_TecnologiaACTI/2012-2014/presentacion_ACTI. pdf

Jiménez, A. (2017). La investigación formativa y los semilleros de investigación en la Facultad de Ciencias y Educación de la Universidad Distrital Francisco José De Caldas. En A. Jiménez, \& otros, Investigación, educación y formación docente: Tercer encuentro de socialización de experiencias investigativas en la Facultad de Ciencias y Educación. 15-26. Bogotá: UD.

Miyahira, J. (2009). La investigación formativa y la formación para la investigación en el pregrado. Med Jeredd.

Montoya, J., \& Peláez, L. (2013). La investigación formativa en sentido estricto Estricto: una Reflexión para Diferenciar su Aplicación en Instituciones de Educación Superior. Entre Ciencia e Ingeniería, 20-25.

Naranjo, B.A. (Julio de 2018). Inclusión Educativa de estudiantes con discapacidad en la UPS sede Guayaquil. En Memoria Académica del Cuarto Congreso Internacional de Ciencia, Tecnología e Innovación de la sociedad. Quito, Ecuador: Abya Yala.
Naranjo, B. A. (2017). Elementos básicos para la inclusión educativa de estudiantes con discapacidad. Boletin REDIPE, 6(9), 132 - 141.

Naranjo, B. A., Naranjo, R. A., Mora, N., \& Huilcapi , D. (2015). La investigación y las TIC para la inclusión educativa de personas con discapacidad: Red RAITI. Guayaquil, Guayas, Ecuador: Educación para la paz, la compresión y el desarrollo de competencias.

OCDE. (2005). Manual de Oslo: GUÍA PARA LA RECOGIDA E INTERPRETACIÓN DE DATOS SOBRE INNOVACIÓN (3era. ed.). Madrid: EUROSTAT.

Parra, C. (2004). Apuntes sobre la investigación formativa. Educación y Educadores.

Paz Saavedra, L. E., Guerrero, J., \& Ortega, V. (2017). Uso de recursos y herremientas TIC para el fortalecimiento de competencias investigativas en el semillero de investigación del programa en Informática de la Universidad de Nariño.

SENPLADES. (2009). Plan Nacional para el Buen Vivir 2009-2013. Quito: SENPLADES.

SENPLADES. (2013). Plan Nacional para el Buen Vivir 2013-2017. Quito: SENPLADES.

Velázquez, M. R. (2017). La investigación formativa Universitaria ¿Posibilidad o necesidad? Quito: Editorial Jurídica del Ecuador.

Vilá Baños, R., Rubio Hurtado, M., \& Berlanga Silvente, V. (2014). La investigación formativa a través del aprendizaje orientado a proyectos: una propuesta de innovación en el grado de Pedagogía. Innovación Educativa, 241-258. 